\title{
Air Ionization and Its Effects on the Health-An Outline of a Research Project
}

\author{
Massimo Scalia ${ }^{1}$, Massimo Sperini ${ }^{1}$, Gualtiero A. N. Valeri ${ }^{2}$ and Vincenzo Isabella Valenzi ${ }^{1,2,3}$ \\ 1. Sezione BEM (Bioelectromagnetism), C.I.R.P.S. (Interuniversity Research Centre for Sustainable Development), "La Sapienza" \\ University, Via Cavour 256, Rome 00184, Italy \\ 2. Department of Chemistry, IURS \\ 3. Director Biomedical Science Department, UNIMEIER, ViaFruà 21/10, Milano 20146, Italy
}

\begin{abstract}
The present paper is aimed to better illustrate the possible association between a particular extremely weak field, the air ions, and the human health response to this solicitation. Methods and instruments for this kind of analysis are here given in the form of a project: a "Ions meter", to reveal and characterize the properties of the air ions, and "APEC 300", an advanced electronic device, both instruments are with a HPR (high power of resolution). APEC 300 can give the response to the possible health effects by measurements of two electrocuteaneous parameter: the potential level and the impedance. The power of resolution of APEC 300 is much higher than that of other usual devices, for instance, the EAV ones (electroacupuncture), this instrument will be completely ready within few weeks.
\end{abstract}

Key words: Air ions, ions meter, electrocutaneous parameters, high resolution, APEC 300, EAV (electroacupuncture).

\section{Introduction}

\subsection{Some Data on Air Ions Properties}

The aerial ions have been studied as environmental important parameter for many decades, and their importance in biological maintenance of the health state is probably due to their spatial electric charge. The presence of the spatial electric charge in the atmosphere is strictly linked also with environmental physics characteristics, meteorology, pollution, climatology and bioclimatology [1-5].

Actually, a complete biophysical model to describe the whole electrical environment in the atmosphere and its biological effects does not exist [6]. We do not yet have the physical laws that rule the complex interdependence of the processes of storms, the maintenance of the natural electric field and the aerial-ion behavior, but all the electric phenomena are

Corresponding author: Vincenzo Isabella Valenzi, M.D., research fields: biometeorology and climate therapy, quantum chemistry and pharmaco-electrodynamics. Email: vincenzo.valenzi@unimeier.eu. usually related to the so called "normal" weather conditions. For instance, the idea of "good weather" is sometimes assumed as the framework for the studies on the effects of altitude climate-therapy of respiratory diseases as well as cardiovascular disorder. This approach will not be correct because the storms and all other weather perturbations represent the way to maintain the equilibrium of the atmosphere system with respect to the spatial electric charge and the natural air ionization, in a feedback mechanism [6]. Thus, a more subtle point of view is needed when assessing the criteria of "good weather" as an equilibrium state in view of a correlation with health conditions.

Anyway, the atmospheric electric charge plays an important role in the climate genesis and the weather evolution. A high correlation exists between the electric conductivity of the low atmosphere and the air ionization, the latter determining the microclimate in which the man lives. The amount of positive and negative ions has a great importance in bioclimatology and affects both the human physiological parameters 
and the behavioral ones, some features of these parameters can be recognized from the Tables 1 and 2 .

\subsection{A Brief Analysis of the Data}

From the reported data it is possible to argue that:

(1) the absolute number of negative and positive ions is higher in outdoor than in indoor, unless in the case of an artificial ionization (laboratory, Table 2 row 2);

(2) a $\mathrm{n}^{-} / \mathrm{n}^{+}$ratio near 1 , the "equilibrium", cannot be showy taken as an index of an environment good to health;

(3) less polluted areas have a higher absolute number of small ions, also indoor. In the latter case the presence of people significantly reduces the small ions concentration;

(4) the subjective perception of "best conditions" seems related to the high number of small ions;

(5) an electrical ionization by means of a flux of big ions can dramatically change the characteristics of the indoor air, surely as a matter of subjective perception.

This list suggests that an association of small ions with good health, beyond the perception of best conditions, can be not arbitrary. On the contrary, there are some considerations in favor of a negative answer to the question: "Is there an impact of the air ions on the human health?".

\subsection{Extremely Weak Fields}

In fact, their "force" seems too weak to produce biological or health effects. It's enough to look at the electric currents induced in our body by air ions: about $(0.01 \div 0.1) \mathrm{nA}\left(1 \mathrm{nA}=1\right.$ nano Ampere $\left.=10^{-9} \mathrm{~A}\right)$, while a wide set of usual electronic devices have an operative range centered on $1 \mu \mathrm{A}(1 \mu \mathrm{A}=1$ micro Ampere $=10^{-6} \mathrm{~A}$ ), that is about $10^{5}$ times higher. An analogue figure is shown by the concentration of air ions: it varies from $10^{-12}$ p.p.m. (part per million) at sea

Table 1 Values of measures of air ions (outdoor).

\begin{tabular}{|c|c|c|c|c|c|c|}
\hline Place & $\mathrm{n}^{-}$Ions & $\mathrm{n}^{+}$Ions & $\mathrm{n}^{-} / \mathrm{n}^{+}$Ratio & $\begin{array}{l}\text { Small Ions } \\
(\mathrm{K} \geq 1)^{1} \\
\%\end{array}$ & $\begin{array}{l}\text { Big Ions } \\
(\mathrm{K}<1)^{1} \\
\%\end{array}$ & $\begin{array}{l}\text { Best }(++++) \\
\text { Worst }(+) \\
\text { perception }\end{array}$ \\
\hline Country at sea level & 303.7 & 251.6 & 1.21 & 88.0 & 12.0 & ++++ \\
\hline Sea, quiet, no wind & 436.7 & 354.4 & 1.23 & 84.7 & 15.3 & +++++ \\
\hline Mountain 2,000 m & 560.3 & 602.5 & 0.92 & 92.0 & 8.0 & +++++ \\
\hline Mountain 2,000 m Phön & 9,632 & 11,79 & 0.81 & 90.0 & 10.0 & +++ \\
\hline Town street no traffic & 120.5 & 108.7 & 1.1 & 66.0 & 44.0 & +++ \\
\hline Town street traffic & 30.8 & 150.4 & 0.2 & 25.0 & 75.0 & + \\
\hline
\end{tabular}

Table 2 Values of measures of air ions (indoor).

\begin{tabular}{lllllll}
\hline Place & $\mathrm{n}^{-}$Ions & $\mathrm{n}^{+}$Ions & $\mathrm{n}^{-} / \mathrm{n}^{+}$Ratio & $\begin{array}{l}\text { Small Ions } \\
(\mathrm{K} \geq 1)^{1} \\
\%\end{array}$ & $\begin{array}{l}\text { Big Ions } \\
(\mathrm{K}<1)^{1} \\
\%\end{array}$ & $\begin{array}{l}\text { Best }(+++++) \\
\text { Worst }(+) \\
\text { perception }\end{array}$ \\
\hline Physic lab & 136.6 & 128.3 & 1.06 & 70.0 & 30.0 & ++++ \\
Physic lab-Ionizer device on & 4,305 & 135.5 & 31.7 & 17.2 & 82.8 & ++ \\
Gymnastic hall no people & 130.3 & 142.6 & 0.91 & 73.0 & 27.0 & +++ \\
Gymnastic hall in use & 13.2 & 32.5 & 0.40 & 42.0 & 58.0 & + \\
Hospital surgery room conditioned & 1.8 & 4.2 & 0.42 & 35.7 & 64.3 & + \\
Classroom no children & 106.3 & 120.5 & 0.88 & 40.1 & 59.9 & +++ \\
Classroom children & 50.3 & 116.5 & 0.44 & 20.2 & 78.8 & ++ \\
Classroom no children near power line & 110.7 & 208.7 & 0.53 & 80.3 & 19.7 & +++ \\
Classroom children near power line & 47.9 & 180.6 & 0.26 & 42.6 & 57.4 & ++ \\
Library in use & 3.7 & 40.8 & 0.09 & 20.6 & 79.4 & + \\
Young cultural center & 12.5 & 0.41 & 30.4 & 15.3 & 84.7 & ++ \\
\hline
\end{tabular}

\footnotetext{
" $\mathrm{K}$ " is the mobility coefficient of different species of ions, bigger than one as their velocity is higher than a fixed value.
} 
level to $10^{-8}$ p.p.m., below an $\mathrm{AC}$ power line, while the concentration of $\mathrm{O}_{3}$ and $\mathrm{SO}_{2}$, responsible for well known effects on the respiratory system, are, respectively, $10^{-2}$ p.p.m. and 1 p.p.m.

In spite of similar considerations, there are a lot of natural phenomena in which a solicitation of a very low intensity is at the basis of a physiological response: the electroreception in elasmobranchs is activated by 1 $\mu \mathrm{V}$; the human eye retina is capable of detecting few photons; a concentration from $10^{-10} \mathrm{M}$ to $10^{-12} \mathrm{M}-\mathrm{a}$ dilution in water equal to that of the air ions at sea level - of the aldosterone or anti-diuretic hormones is capable of saving the sodium request of the renal tubules in our body, and so on.

In any case, many scientific works have tried to give an answer if the air ion, weak or less weak is the intensity of their solicitation, have some significance for the behavior of biological systems and the state of human health, in the past and also in the more recent years (see, for instance, Refs. [7-17]).

Another issue has come to the attention since "the golden age of house-dust mites is over": the "new" indoor contaminants - molds, bacteria and their metabolites - have run the gauntlet to be investigated as attacking human health. The variation of the air ion levels has been hypothesized as a stress factor acting on children [18], as well as the correlation between the change of meteorological conditions and the onset of myocardial infarction [19]. Many relevant chronobiological studies about the influence of high altitude on some physiological parameters (pressure of blood, heart rate, circadian and infradian cardiovascular rhythms, arterial stiffness and neurocardio-pulmonary function, etc.), also in relationship with the ageing of populations, are quoted in bibliography [20].

\section{Materials and Methods}

\subsection{Measuring Electrocutaneous Parameters}

A latere of the previous proposed, and partly experienced, methodologies, another way to possibly have more direct results in order to assess the effect of the air ions, or of other pollutants, on the human health is measuring the electrocutaneous parameters: the impedance and the potential level, and their variations in time. In the case of the air ions, this implies we have previously characterized the local environment-outdoor, indoor - by measuring the fundamental parameters of the air ions, that is, their current (the number of ions) and their mobility.

Referring to the methodology of electrocutaneous measures, the role of the skin has to be emphasized: it cannot be more thought as a passive element of our organism, since it is deeply correlated with almost all organs of our interior (lungs, heart, respiratory organs, liver, etc.). It's a long time that the skin has become a protagonist, surely in Western Countries, since when Reinhold Voll launched his electroacupuncture (EAV) as a diagnostic method in Medicine [21]; a method still practiced everywhere in the world [22]. Apart from the EAV method, that needs special electrodes point shaped, and a special skill, mainly the measures of the potential level, but also of the impedance of the skin, or of the derma, could be done by means of the usual electrodes and could give important information on the health state, also for a single organ, whatever is the nature of the stress acting on the body; even though these measures are not taken in correspondence to the dermatomes (dermatomera) [23].

Reliable indications on the health in children, or in other critical groups to be tested, could be obtained, restricting oneself only to measurements of the potential level of the skin - a measure without any kind of stimuli, just like an EEG or ECG, that does not require to be allowed by sanitary authorities, and comparing the results, specially the Fourier spectrum of the potential, in the two different environments: at sea level in an urban context, at altitude in a not polluted area.

This methodology demands new techniques of measurement, quite more advanced than the EAV ones or the other techniques usually applied to the today 
scientific research. In fact, due to the already underlined smallness of the values, these proposed measurements-air ions, electrocutaneous parameters-require electronic instruments characterized by a very high power of resolution.

\subsection{HPR (High Power of Resolution) Devices: "Ions Meter" and "APEC 300"}

About all this matter we have got ready to a research project, the general purpose of which is to investigate biological and health effects as consequences of the climate at high altitude.

The scope is to obtain results that better show the correlation among the air ions, or other climate characteristics or polluting factors, and the biological systems and, mainly, the response of the human health, by means of new techniques of measurement.

In fact, the validity of the correlation strongly depends on the accuracy of measurements, which, in turn, require a high power of resolution in determining the parameters characterizing the air ions as well as determining cutaneous potential levels or impedances. Thus, an objective that coordinates with the general aim is to completely realize the two HPR electronic instruments that are necessary for reliable measures: an "Ions meter" and an electrocutaneous parameters analyzer (APEC 300).

HPR is requested in both cases by the smallness of the intensities of what one wants to measure; in the case of the air ions, which act as an external stimulus, a more precise association with a health response requires an air ions measurement apparatus capable of appreciating a $10 \mathrm{fA}^{2}$ current, that is, tens of ions.

On the other hand, the electrocutaneous parameters need a sensibility of $0.01 \mathrm{nV}\left(1 \mathrm{nV}=1\right.$ nano Volt $=10^{-9}$ Volt) on a measure of $0.1 \mathrm{nV}$, which can be the values characteristic for the response in tension in the environment of many industrial areas.

The first device, the "Ions meter", has been already projected and realized. The tuning of this instrument,

\footnotetext{
$2 \mathrm{fA}$ : femtoAmpere, $1 \mathrm{fA}=10^{-5} \mathrm{nA}$
}

necessary to perform those profiles of the research that demand HPR, will require a considerable amount of time to be completed; nevertheless, many measures useful for the proposed research project can be done by the "Ions meter" at its present stage.

The second one, APEC 300, is the apparatus to measure the electrocutaneous parameters at the required level of resolution; it needs only a few weeks to be completely tested and verified. Regarding the cutaneous response of the human organism, the values of measure vary in the milliVolt range, but the nanoVolt range is requested in order to appreciate the electric fields emitted by the skin superficial stratum. In fact, the variations of these electric fields, and their frequency spectrum, are the signals of a physiological behavior of the human organism or the onset of some pathology. The kind of pathology or benefit can be revealed by the characteristics of the frequency spectrum of the response; that can be better assessed through an examination that should operate at cell culture levels. This latter analysis needs a higher power of resolution, up to $0.1 \mathrm{nV}$.

Some experiments, which have been performed on mice living in an urban context, have suggested to check their behavior at altitude (Briançon) and then extend to humans this kind of test, by the means of the project in progress: a climate cure for children, with doses of 1 week, 1 month, before Christmas break and, then, after the holidays.

This objective will imply direct experiments at high altitude with children and possibly with other "critical" groups (ageing people, pregnant women); the minimal time of exposition will be one week. The physiological data taken at altitude will be compared with the values of the same parameters, registered at sea level. The experimental protocol will be derived from the literature and the previous experiences of the researchers of the group.

Useful information will be obtained by an experiment with mice exposed to high altitude climate, to be compared with the one already realized at sea 
level in an urban context, this experiment will precede the ones with human beings.

\section{Results and Discussion}

Some interesting data for air ions in mountain climate, as a new environmental parameter, have been gathered in the early Ninety $[24,25]$. They have been recently republished in a review [26]; the methods of measurements, models and correlations with human health have been republished in another review that collects a number of papers [27].

Now, in the present project, the analysis of a possibly different behavior, at sea level and at altitude, could be performed recurring to the two already mentioned devices_- "Ions meter" and APEC 300 - realized in the framework of the researches led by the Section BEM of C.I.R.P.S. ${ }^{3}$. Since the technology of high resolution instruments is very difficult to find on the market, it has become mostly an apanage of military laboratories and projects, the researchers involved in the project have overcome this situation by designing not only the whole apparatuses but also the main components of each device.

Further, another relevant information can be obtained from carrying out tests also on human cell cultures, as markers of a specific action of the air ions; the latter are in fact, due to the modality provided for the experiments, the only relevant external influence which could act on cells. The recourse to the human cells as a test has been already experimented in a pair of campaigns aimed to an electromagnetic characterization of the environment [28].

It's worthwhile to note that in literature, most of all scientific researches mention measures of impedance or resistance of the biological objects, actually ignoring the role of the cutaneous potential level. On the contrary, as it has been previously emphasized, the values of the potential level can precisely inform about the deviation from the homeostasis of a tissue or an

${ }^{3}$ CIRPS-Centro Interuniversitario di Ricerca per lo Sviluppo Sostenibile (Interuniversity Research Centre for Sustainable Development), "La Sapienza" University, Rome (Italy). organ, reducing to a simple measure of a parameter the timely knowledge of which in Medicine is the supervene of a functional disturb.

Our first measurements of electrocutaneous potential level and of its Fourier spectrum have shown a significance that will make unjustifiable to neglect it; the Fourier spectrum seems to characterize the specific response of every tested biological object.

\section{Conclusions}

The challenge is to verify and improve the results already known, and also to get a better control on the phenomenological aspects. The data so acquired will be able to confirm many current hypotheses or to advance new ones, with the double advantage of an experimental check and of being able to build more well founded models or theories in this domain, and eventually to acquire a deeper insight of many biophysical mechanisms concerning the health state.

\section{Reference}

[1] Rycroft, M. J., Israelsson, S., and Price, C. 2000. "The Global Atmospheric Electric Circuit, Solar Activity and Climate Change." Journal of Atmospheric And Solar-Terrestrial Physics 62: 1563-76.

[2] Panneerselvan, C. 2010. "Instrumentation for the Surface Measurements of Atmospheric Electrical Parameters at Maitri, Antarctica: First Results.” Earth Planet Space 62: 545-9.

[3] Crowley, J. M. 2011. "The Fair-Weather Atmosphere as a Power Source." In Proceedings of ESA Annual Meeting on Electrostatic, 1-14.

[4] Bhattacharya, A. B. 2011. "On Some Characteristic of Tropical Atmospheric and Cosmic Radio Noise." International Journal of Engineering Science and Technology 3 (7): 5475-86.

[5] Skromulis, A., and Noviks, G. 2012. "Atmospheric Light Air Ion Concentrations and Related Meteorological Factors in Rezekne City, Latvia." Journal of Environmental Biology 33: 455-62.

[6] Scalia, M., Pulcini, F., and Sperini, M. 2015. Elementi di teoria: lo stato di salute. Roma: Andromeda.

[7] AA.VV. 1969. In II Simposio Annuale di Bioclimatologia Medica, 6-10.

AA.VV.1971. In IV Simposio Annuale di Bioclimatologia Medica, Villa Olmo Como, 782-5.

[8] Gilbert, G. O. 1973. "Effect of Negative Air Ions upon 
Emotionality and Brain Serotonin Levels in Isolated Rats." International Journal of Biometeorology 17 (3): 267-75.

[9] Gualtierotti, R. 1975. "Azioni biologiche della ionizzazione dell'aria. Rassegnadellaletteratura." In Lacustrine Environment. Methods of Study, 246-78.

[10] Krueger, A. P., Reed, E. J., Brook, K. P., and Day, M. B. 1975. "Air Ion Action on Bacteria." International Journal of Biometeorology 19 (1): 65-71.

[11] König, H. L., and Krueger, A. P. 1981. Biological Effects of Environmental Electromagnetism. New York: Springer-Verlag.

[12] Lambert, J. F., Olivereau, J. M., and Truong-Ngoc, A. 1981. "Influence of Artificial Air Ionization on the Electroencephalogram of the Awake Rat." International Journal of Biometeorology 25 (1): 71-5.

[13] Reiter, R. 1985. "Present State of Knowledge Regarding Biological Effects of Magnetic, Electric and Electromagnetic Fields, as well as of Natural Radioactivity in Indoor Air." International Journal of Biometeorology 29 (2): 115-37.

[14] Kinne, S. M. 1997. "A Public Health Approach to Evaluating the Significance of Air Ions." Master of Public Health thesis, University of Texas, Health Science Center at Houston School of Public.

[15] Otsuki, T. 2009. "The Biological Effects of Negatively-Charged Indoor Air Conditions." Kawasaki Medical Journal 35 (3): 205-14.

[16] Kung, C.-C. K., and Pollack, G. H. 2014. "Effect of Atmospheric Ions on Interfacial Water." Entropy 16: 6033-41.

[17] Wiszniewski, A., Suchanowski, A., and Wielgomas, B. 2014. "Effects of Air-Ions on Human Circulatory Indicators." Pol. Journal Environmental Study 23 (2): 521-31.

[18] Fornof, K. T., and Gilbert, G. O. 1988. "Stress and Physiological, Behavioral and Performance Patterns of Children under Varied Air Ion Levels." International Journal of Biometeorology 32: 260-70.

[19] Stoupel, E. 1995. "Relationship between Suicide and Myocardial Infarction with regard to Changing Physical Environmental Conditions." International Journal of Biometeorology 38: 199-203.

[20] Watanabe, Y. 2007. "Chronobiological Assessment of Circadian Rhythm of Blood Pressure and Heart Rate at High Altitude on Mt. Fuji." In Proceedings of the 2nd World Congress of Chronobiology, 88.

Otsuka, K. et al. 2005. "Effect of Aging on Blood Pressure in Leh, Ladakh, a High-Altitude (3524 m) Community, by Comparison with a Japanese Town." Biomed \& Pharmacother 59 (Suppl 1): 54-7.

Kawasaki, T. et al. 2013. “Astro-glocal Spatially and
Temporally (Global And Local) Comprehensive Health Watch, Especially at High Altitude." In Proceedings of International Conference, Space Weather Effects in Humans, 539-50.

[21] Leonhardt, H. 1980. Fundamentals of Electroacupuncture According to Voll. Uelzen: Medizinisch Literarische VergsgesellschaftmbH.

Kenion, J. N. 1983. Modern Techniques of Acupuncture. A Practical Scientific Guide to Electro-Acupuncture. New York: Thorsons Publishers Inc.

[22] Casimiro, L. 2009. Acupuncture and Electroacupuncture for the Treatment of Rheumatoid Arthritis. The Cochraine Collaboration: Wiley \& Sons.

Dubounet, D. 2009. Electroacupuncture, the Scientific Treatise. Paris: Maitreya.

Quispe-Cabanilas, J. G. et al. 2012. "Impact of Electroacupuncture on Quality of Life for Patients with Relapsing-Remitting Multiple Sclerosis under Treatment with Immunomodulators: A Randomized Study." Complementary and Alternative Medicine 12: 1-9.

Sandra, S.-L. 2012. Electroacupuncture and Stimulatory Frequencies for Analgesia. Curitiba: Institute Brasileiro de Therapias e Ensimo (IBRATE).

[23] Bradshaw, L. A. 1997. "Correlation and Comparison of Magnetic and Electric Detection of Small Intestinal Electric Activity." American Journal of Physiology 272: 1159-67.

Glickman, Y. A. et al. 2003. "Electrical Impedance Scanning: A New Approach to Skin Cancer Diagnosis." Skin Research and Technology 9: 262-8.

Kyle, U. G. 2004. "Bioelectrical Impedance Analysis-Part I: Review of Principles and Methods." Clinical Nutrition 23: 1226-43.

Kyle, U. G. 2004. "Bioelectrical Impedance Analysis-Part II: Utilization in Clinical Practice." Clinical Nutrition 23: 1430-53.

Ahmed, S. et al. 2009. Transepithelial Electrical Resistance on Cell Cultures for In Vitro Toxicity Testing of Water Samples. Swiss Scientific Initiative in Health Report.

Itseksen, A. et al. 2010. "Measurement of Electrical Resistance of Dermal-Visceral Zones as A Diagnostic Tool for Gynecological Disorders." The Israel Medical Association Journal 12: 334-7.

Boucsein, W. et al. 2012. "Publication Recommendations for Electrodermal Measurements." Psychophysiology 49: 1017-34.

[24] Marinelli, F., Sperini, M., and Scalia, M. 1990. "Misure di ionizzazione dell'aria in ambiente alpino." In Associazione Italiana di Aerobiologia-IV Congresso Nazionale Aereosol Biologico (sorgenti, dispersione 
impatto).

[25] Marinelli, F., Sperini, M., Scalia, M., and Murri, A. 1991. "The Air Ionization: A New Environmental Parameter." In International Symposium: Water, Atmosphere and Soil, a New Environmental Deal.

Marinelli, F., Sperini, M., Scalia, M., and Murri, A. 1991. "Un importante parametro in climatologia montana. La ionizzazione dell'aria." In Proceedings of International Medical Congressof Mountain Climatology.
[26] Scalia, M., Sperini, M., Marinelli, F., and Valenzi, V. 2013. Ioni aerei e salute umana. Roma: Andromeda.

[27] Scalia, M., Sperini, M., and Guidi, F. 2014. Effetti biologici degli ioni aerei misure e modelli. Roma: Andromeda.

[28] Scalia, M., and Sperini, M. 2013. "Monitoraggio elettromagnetico ambientale". Convenzione ARPA Lazio-CIRPS. http://www. cirps.it/news/13-news-21, www.arpalazio.gov. it/servizi/eventi/index.htm?page $=6$ 